\title{
Guest Reviewers List
}

(C) Canadian Anesthesiologists' Society 2012

On behalf of the Editorial Board, the Editor-in-Chief acknowledges, with special thanks, the expertise of the following individuals who served as Guest Reviewers from July 1, 2011 to June 30, 2012.

aan het Rot, Marije / GRONINGEN Afshari, Arash / COPENHAGEN Angle, Pamela / toronto Ansermino, J. Mark / VANCOUVER Ansley, David / VANCOUVER Archer, David / CALGary Asai, Takashi / MORIGUCHI Asenjo, Juan-Francisco / MONTREAL Avram, Michael J. / CHICAGO

Badner, Neal / LONDON Bagshaw, Sean M. / EDMONTON Baxter, Frederick J. / HAMILTON Beloeil, Hélène / LE KREMLIN-BICÊTRE Benhamou, Dan / PARIS Billard, Valérie / VILLEJUIF Boet, Sylvain / TORONTO Bosey, M. Gregory / St. CATHARINES Bould, M. Dylan / otTawa Bourgain, Jean-Louis / VILLEJUIF Bourke, Michael E. / OTTAWA Bracco, David / MONTREAL Bransborg, Birgitte / AARHUS Brennan, Tim / IOWA Brown, Karen A. / MONTREAL Bussières, Jean / QUEBEC CITY Byrick, Robert / TORONTO Cahill, Cathy / KINGSTON Calvert, John W. / ATLANTA Cannon, John E. / WinNIPEG Carli, Franco / MONTREAL Carvalho, Jose C.A. / TORONTO
Chan, Vincent / TORONTO

Chaput, Alan J. / OTTAwA Chartrand, Daniel / MONTREAL Choi, Peter / vancouver Chung, Frances / TORONTO Clarke, Hance / TORONTO Combes, Xavier / PARIS Cooper, Richard M. / TORONTO Cox, Robin G. / CALGaRY Craen, Rosemary A. / LONDON Crosby, Edward / OTTAWA Crystal, George J. / CHICAGO Cuillerier, Daniel J. / LONDON Dain, Steven / LONDON Davies, Jan M. / CALGARY Denault, André / MONTREAL Dexter, Franklin / IOWA Djaiani, George / TORONTO Doherty, Dermot / otтawa Dupuis, Jean-Yves / OTTAWA Ecoffey, Claude / RENNES Eipe, Naveen / OTTAwA Eisenkraft, James B. / NEW YORK El-Beheiry, Hossam / TORONTO Elwood, Tom / TACOMA

Estebe, Jean-Pierre C. / SEATtLE Evans, Holly / OTTAwA Faccenda, Kathryne A. / EDMONTON Fayad, Ashraf / OTTAWA Fergusson, Dean A. / OTTAwA Finegan, Barry A. / EDMONTON
Froese, Alison B. / KInGSTON Fuchs-Buder, Thomas / NANCY Funk, Duane / winNIPEG Ganapathy, Sugantha / LONDON George, Ronald B. / HALIFAX Gilron, Ian / KINGSTON Goldstein, David / KINGSTON Granton, Jeff / HAMILTON Grégoire, Marie-Claude / HALIFAX Guay, Joanne / MONTREAL Gupta, Sanjiv / OTTAWA Habib, Ashraf S. / DURHAM Hall, Richard I. / HALIFAX Hamstra, Stanley J. / OTTAwA Hashimoto, Kenji / CHIBA Heindl, Bernhard / MUNICH Holtby, Helen / TORONTO Houston, Patricia / TORONTO Hung, Orlando / HaLIFaX Jacka, Michael / EDMONTON Jones, Philip / LONDON Joshi, Girish / DALLAS Kleine-Brueggeney, Maren / BERN Kulkarni, Pradeep R. / EDMONTON Labrecque, Pascal / QUEBEC CITY Laffey, John G. / GaLway Lavand'homme, Patricia M. / BRUSSELS Lavigne, Gilles / MONTREAL Lavoie, Josée / MONTREAL Law, Adam / HALIFAX LeDez, Ken / Sт. JOHN's 
Lemoine, Sandrine / CAEN Lentschener, Claude / PARIS Lerman, Jerrold / BUFFALO Lessard, Martin R. / QUEBEC CITY Ling, Elizabeth / HAMILTON London, Martin / SAN FRANCISCO Manninen, Pirjo / TORONTO Martin, René / SHERBROOKE McCartney, Colin / TORONTO McCluskey, Stuart / TORONTO McIsaac, Daniel / OTTAWA McKay, William P. / SASKATOON McKeen, Dolores M. / HALIFAX Merchant, Richard N. / vANCOUVER Mertes, P. Michael / NANCY Minkovich, Leonid / TORONTO Murkin, John / LONDON Murphy, Michael / EDMONTON Mutch, W. Alan C. / WINNIPEG Naguib, Mohammed / CLEVELAND Naik, Viren / TORONTO Nathan, Howard / OTTAwA Neilipovitz, David T. / OTTAWA
Neira, Victor / OTTAWA

Neustein, Steve / NEW YORK Parlow, Joel / KInGSTON Paul, James / HAMILTON Penning, John P. / otTawa Perlas, Anahi / toronto Punja, Alim / otTAWA Raemer, Dan / BOSTON Ralley, Fiona E. / LONDON Raymer, Karen / HAMILTON Riazi, Sheila / TORONTO Richebé, Philippe / SEATTLE Rigamonti, Andrea / TORONTO Ripart, Jacques / NIMES Romaschin, Alex / TORONTO Sawka, Andrew / vanCOUver Servin, Frédérique / PARIS Shehata, Nadine / TORONTO Sheppard, Shane / SASKATOON Singh, Sudha / LONDON Slinger, Peter / TORONTO Sohmer, Benjamin / OTTAwA Splinter, William M. / отTAWA
Stafford-Smith, Mark / DURHAM Sullivan, Michael / NEWMARKET Tait, Gordon / TORONTO Taylor, Katherine / TORONTO Tran, De Q.H. / MONTREAL Turkstra, Timothy / LONDON Turner, Kim / KINGSTON Van der Linden, Philippe / BRUSSELS van Klei, Wilton A. / UTRECHT van Vlymen, Janet / KINGSTON Veroux, Massimiliano / CATANIA Wald, Richard A. / VANCOUVER Warriner, Brian / VANCOUVER Wasowicz, Marcin / TORONTO Wassermann, Jeff / TORONTO Whyte, Simon / VANCOUVER Wijeysundera, Duminda N. / TORONTO Withington, Davinia E. / MONTREAL Wong, Anne / HAMILTON Wong, Jean / TORONTO Xia, Zhengyuan / HONGKONG Zaballos, Matilde / MADRID Zaugg, Michael / EDMONTON 